\title{
Implementation of a Low-Complexity Frame-Start Detection Algorithm for MIMO Systems
}

\author{
David Perels, Christoph Studer, and W. Fichtner \\ Integrated Systems Laboratory, ETH Zurich, Switzerland \\ \{perels,studer,fw\}@iis.ee.ethz.ch
}

\begin{abstract}
Multiple-input multiple-output (MIMO) communication systems require well-designed synchronization schemes at the receiver to meet stringent $Q 0 S$ requirements. In particular, OFDM modulation is very sensitive to timing synchronization errors which incur inter-symbol interference. This paper describes a frame-start detection algorithm, which relies on received signal power increase and does not require any special properties of the transmitted signal. The performance is analyzed and then verified through simulations in a MIMO-OFDM system. Finally, a lowcomplexity FPGA implementation of the presented algorithm is described in detail.
\end{abstract}

\section{INTRODUCTION}

Multiple-input multiple-output (MIMO) technology has gained considerable attention throughout the last years [1]. Orthogonal frequency division multiplex (OFDM) modulation technique allows efficient communication over frequency selective wireless channels. The combination of both techniques constitutes the basis for many upcoming standards, such as IEEE $802.11 \mathrm{n}$ or IEEE 802.16e.

Almost any MIMO-OFDM wireless local area network (WLAN) system is frame-based. The performance of the MIMO receiver strongly depends on the ability to synchronize on the incoming frames. One important aspect of the synchronization is frame-start detection. So far, most of the proposed algorithms base on the periodicity of the incoming signals, e.g. [2], [3], [4]. However, if the incoming signal is not periodic or an automatic gain controller (AGC) is present in the system, proper frame-start detection becomes difficult. If the signal is periodic it is possible to acquire frame timing at the end of the periodic signal which incurs significant hardware costs. To this end, an implementation of a signal-power based frame-start detector has been presented in [5]. There, the signal power is evaluated independently on each receive antenna with subsequent evaluation of all antenna results. However, this strategy does not properly exploit the degrees of freedom offered by multiple receive antennas.

Contributions: In this paper we describe an implementation of a low-complexity frame-start detection algorithm for MIMO systems. The detection is based on the observation of instantaneous signal power, which turns out to be a promising approach in the presence of multiple receive antennas. Additionally, no special properties (e.g. periodicy etc.) of the transmitted signals are required. The performance of the frame-start detection algorithm is analyzed and simulations in a MIMO-OFDM system are performed. Finally, a low-complexity architecture for an FPGA implementation is described in detail.
Notation: Discrete sample instants are denoted by $k$. The probability density function (PDF) of a random variable $X$ is $p(x)$ and the corresponding cumulative distribution function (CDF) is denoted by $F(x)$. The mean of random variable $X$ is given as $E[X]$. The probability of an event $\mathcal{E}$ is denoted by $\operatorname{Pr}[\mathcal{E}]$. The complex-valued Gaussian distribution with zero mean and variance $\sigma^{2}$ is denoted by $\mathcal{C N}\left(0, \sigma^{2}\right)$. The Gamma function [6] is $\Gamma(x)=\int_{0}^{\infty} y^{x-1} e^{-y} d y$ and $\Gamma(x, a)=$ $\int_{a}^{\infty} y^{x-1} e^{-y} d y$ denotes the upper-incomplete Gamma function, respectively. The PDF of a Gamma-distributed random variable $\sim G(a, b)$ is given by

$$
p_{\text {Gamma }}(x)=\left\{\begin{array}{cl}
\frac{x^{a-1} e^{-\frac{x}{b}}}{\Gamma(a) b^{a}} & x \geq 0 \\
0 & x<0
\end{array}\right.
$$

Outline: The remainder of this paper is organized as follows: Section II describes the system model and the framestart detection algorithm is presented and analyzed Section III. Simulation results of a $4 \times 4$ MIMO-OFDM system are presented in Section IV and Section V describes a lowcomplexity FPGA implementation.

\section{SySTEM MOdEL}

Consider a sampled baseband-equivalent MIMO system with $M_{R}$ receive and $M_{T}$ transmit antennas, as depicted in Fig. 1. We herein assume fading and non-fading channel scenarios. Each receive antenna obtains a superposition of $M_{T}$ transmit signals. Thermal noise, denoted by $n_{i}[k]$ with $i=1, \ldots, M_{R}$, disturbs the received signal and is assumed to be i.i.d. $\sim \mathcal{C N}(0,1)$. Each receive antenna obtains $r_{i}[k]$. We assume that the transmitter does not send any signals during $k<k_{0}$ and thus, only thermal noise is present at the receiver. At $k=k_{0}$ the transmitter starts to send a frame and therefore, the receiver obtains a superposition of transmit signals and thermal noise for $k \geq k_{0}$.

\section{Frame-Start Detection Algorithm}

\section{A. Algorithm}

The main idea of the proposed frame-start detection algorithm is to compare the instantaneous power increase on all receive antennas to a long-term power estimate and then, decides for an estimated frame-start instant $\hat{k}_{0}$. To this end, the detector calculates the instantaneous signal power per receive antenna by computing the squared absolute value of all $M_{R}$ 


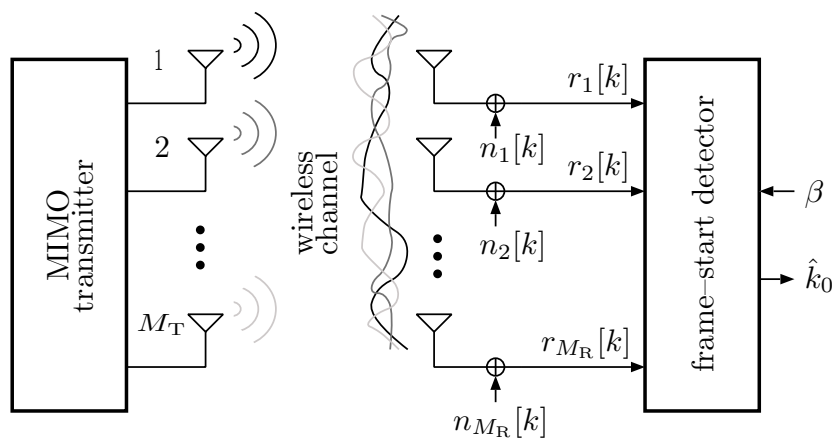

Fig. 1. MIMO-OFDM system model with $M_{R}$ receive and $M_{T}$ transmit antennas (only signals relevant for the frame-start detector are shown).

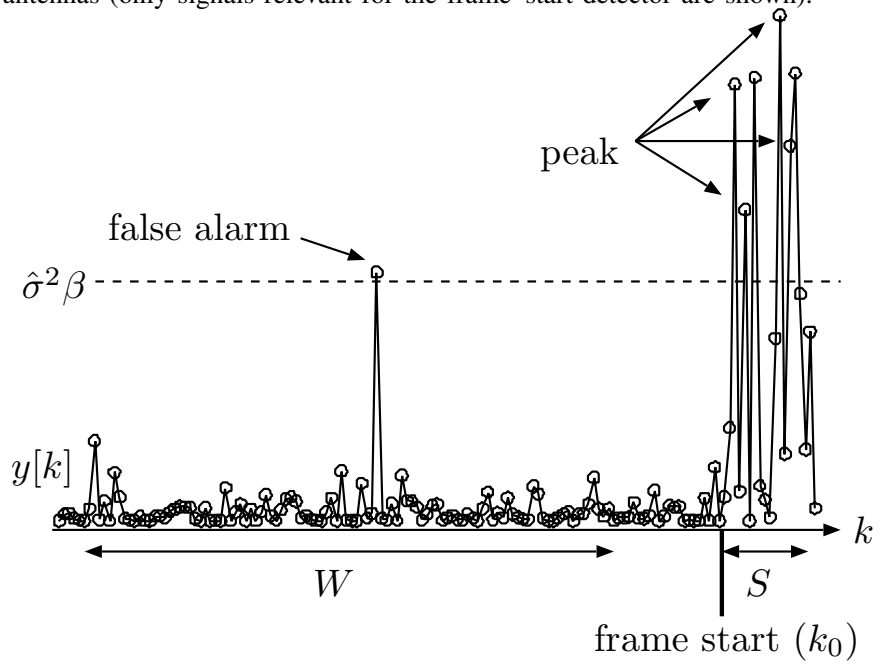

Fig. 2. A possible signal $y[k]$ at the input of the comparator. The window for false peak detection is $W$ samples and $S$ samples for the frame detection. Too many false peaks may inhibit the detection of a frame-start.

complex-valued baseband samples $\left|r_{i}[k]\right|^{2}$. The sum of all instantaneous signal powers is denoted by

$$
y[k]=\sum_{i=1}^{M_{R}}\left|r_{i}[k]\right|^{2} .
$$

A power estimator (PEST) unit computes the average receive power $\hat{\sigma}^{2}$ based on many observed $y[k]$, i.e.,

$$
E[y[k]]=\hat{\sigma}^{2}=M_{R} \quad k<k_{0} .
$$

Then, a comparator unit decides whether the instantaneous power $y[k]$ is larger than a given threshold. A single peak is detected if $y[k]$ exceeds $\beta \hat{\sigma}^{2}$ (and $\beta>0$ ) and $f[k]$ indicates a peak by setting $f[k]=1$. Then, a frame-start can be confirmed by a finite-state machine (FSM) by the detection of a sustained power increase $\hat{\sigma}^{2}$, which is characterized by a series of detected peaks. Finally, the FSM provides an estimate $\hat{k}_{0}$ of the true frame start. Subsequently, other synchronization circuitry may measure the periodicity of the received signal (e.g. [2]) to indicate the presence of a short preamble signal in IEEE 802.11a, for example.

\section{B. Performance Analysis}

We divide the performance analysis of the frame-start detection algorithm into three parts. In the first part the performance measures are introduced. Then, the false peak and false alarm probability are derived without any simplifications. In the third part, the missed frame probability is analyzed using a simplified system model.

1) Performance Measures: The performance of the proposed frame-start algorithm is characterized by three probability measures. The peak probability indicates the likelihood that a peak has falsely been detected and no frame has been transmitted, i.e.,

$$
P_{\mathrm{p}}[k]=\operatorname{Pr}\left[y[k]>\hat{\sigma}^{2} \beta\right] \quad k<k_{0} .
$$

The false alarm probability denotes the probability that over a window of $W$ samples at least one false peak ${ }^{1}$ has been detected, i.e.,

$$
P_{\text {fa }}=1-\left(1-P_{\mathrm{p}}[k]\right)^{W} \quad k<k_{0},
$$

and $W$ denotes a window width, where no false peak can be tolerated. This parameter should be chosen system dependent. For example in IEEE 802.11a, $W$ should be larger than several OFDM symbols. Finally, the probability of a missed frame is denoted by

$$
P_{\mathrm{mf}}=\left(1-P_{p}[k]\right)^{S} \quad k \geq k_{0},
$$

where $S$ denotes a small window, where at least one peak has to be detected. Note that $S$ has to be chosen small, since the estimated frame-start instant $\hat{k}_{0}$ should not be much later than $k_{0}$. In OFDM systems $S$ has to be chosen smaller than $N_{\mathrm{c}}-L$ (where $N_{c}$ and $L$ denote the cyclic-prefix length and channel length, respectively) to allow inter-symbol interference free reception.

2) False Alarm Probability: If the transmitter does not send any signal (i.e., $k<k_{0}$ ), $r_{i}[k]$ does only contain thermal noise. Thus,

$$
y[k]=\sum_{i=1}^{M_{R}}\left|n_{i}[k]\right|^{2} \quad k<k_{0},
$$

which is a sum of absolute-squared Gaussian random variables. The PDF of (7) corresponds to a Gamma distribution [6]:

$$
y[k] \sim G\left(M_{R}, 1\right) \quad k<k_{0}
$$

Thus, the peak probability (4) is equal to

$$
P_{\mathrm{p}}[k]=\frac{\Gamma\left(M_{R}, \hat{\sigma}^{2} \beta\right)}{\Gamma\left(M_{R}\right)} \quad k<k_{0} .
$$

Note that $P_{\mathrm{p}}[k]$ only depends on the number of receive antennas and $\hat{\sigma}^{2} \beta$. Then, the false peak detection probability within a given window $W$ can be computed by using (5).

The key design parameter of the proposed frame-start detection algorithm is the threshold parameter $\beta$, which directly influences the false alarm rate $P_{\mathrm{fa}}(5)$. The system designer can specify a maximum tolerable false alarm rate and a number of receive antennas $M_{R}$, which is sufficient to compute the corresponding $\beta$. Tab. I shows the choice of the threshold for

\footnotetext{
${ }^{1}$ Here it is important to note that false alarms have to be kept as low as possible as it may be possible that a frame-start can be missed if the FSM is overloaded by false peaks.
} 
TABLE I

CHOICE OF THE THRESHOLD PARAMETER $\beta(W=8000)$

\begin{tabular}{|l||c|c|c|c|}
\hline$P_{\mathrm{fa}}$ & $M_{R}=1$ & $M_{R}=2$ & $M_{R}=3$ & $M_{R}=4$ \\
\hline \hline $10 \%$ & 11.2 & 7.0 & 5.4 & 4.6 \\
\hline $1 \%$ & 13.6 & 8.2 & 6.3 & 5.3 \\
\hline
\end{tabular}

false alarm rates of $10 \%$ and $1 \%$ depending on $M_{R}$. Note that $\beta$ should preferably be chosen as a power of two in order to reduce hardware complexity and to avoid multipliers.

3) Missed Frame Probability: To analyze the missed frame probability (6), two simplifications are used to model a frame start. We assume a simple random model and do not impose any special properties (e.g. periodicy) on the transmit signal. First, we assume that if $k \geq k_{0}$ a sudden power increase of the received noise $n_{i}[k]$ occurs, as illustrated in Fig. 2. Additionally, the power increase is assumed to be equal on all receive antennas. Hence, the received signal can be described as

$$
r_{i}[k]=I[k] n_{i}[k] \quad i=1, \ldots, M_{R},
$$

where $I[k]$ is defined as

$$
I[k]= \begin{cases}1 & k<k_{0} \\ \sqrt{\Delta P} & k \geq k_{0}\end{cases}
$$

and $\Delta P$ corresponds to the power increase per receive antenna. This sudden signal increase at sample instant $k=k_{0}$ by $\Delta P$ at the input of the antennas affects the peak probability. The signal power increase can equally be expressed as a sudden reduction of the threshold $\hat{\sigma}^{2} \beta$ by the factor $1 / \Delta P$ at $k \geq k_{0}$. Thus, analogous to (9), the peak probability after a frame-start $\left(k \geq k_{0}\right)$ can be computed as

$$
P_{\mathrm{p}}[k]=\frac{\Gamma\left(M_{R}, \frac{\hat{\sigma}^{2} \beta}{\Delta P}\right)}{\Gamma\left(M_{R}\right)} \quad k \geq k_{0} .
$$

The probability of a missed frame $P_{\mathrm{mf}}$ depends on the instantaneous power increase and can be obtained by combining the results of (12) with (6). In Fig. 3 the frame-miss probabilities for this signal model are plotted for several power increases $\Delta P$. The performance gain by using multiple receive antennas is clearly visible in this plot.

\section{Simulation Results}

To predict the real-world performance of the proposed frame-start detection algorithm a $4 \times 4$ MIMO-OFDM system based on IEEE 802.11a has been simulated. The signal to be detected corresponds to the short preamble based on IEEE 802.11a [7], where the active tones are distributed over all transmit antennas as described in [8]. Channels with different numbers of taps $L$ and Rayleigh-fading have been simulated. To allow a better comparison between the different channel scenarios, the detection window $S$ has been set to 5 . The simulations are done by sweeping the signal-to-noise ratio (SNR), starting from $\mathrm{SNR}_{\mathrm{dB}}=1$ in $1 \mathrm{~dB}$ steps. Note that $\mathrm{SNR}_{\mathrm{dB}}$ and the power increase $\Delta P\left(\right.$ at $\left.k \geq k_{0}\right)$ are related by

$$
\Delta P=10^{\frac{\mathrm{SNR}_{\mathrm{dB}}}{10}}+1
$$

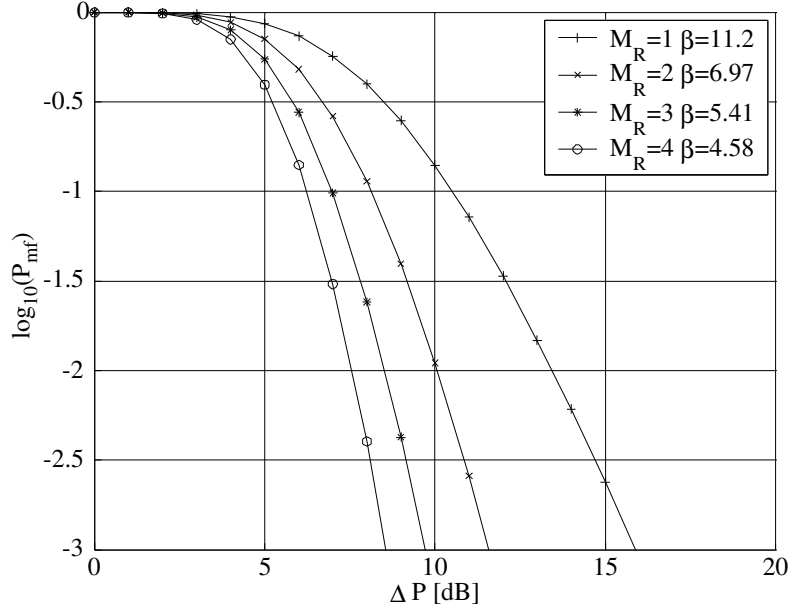

Fig. 3. Approximated effect of multiple receive antennas $\left(M_{R}=1,2,3,4\right)$ on missed frame rate for different power increases $\Delta P$. The false alarm rate $P_{\mathrm{fa}}$ has been fixed to $10 \%$ and $W=8000$.

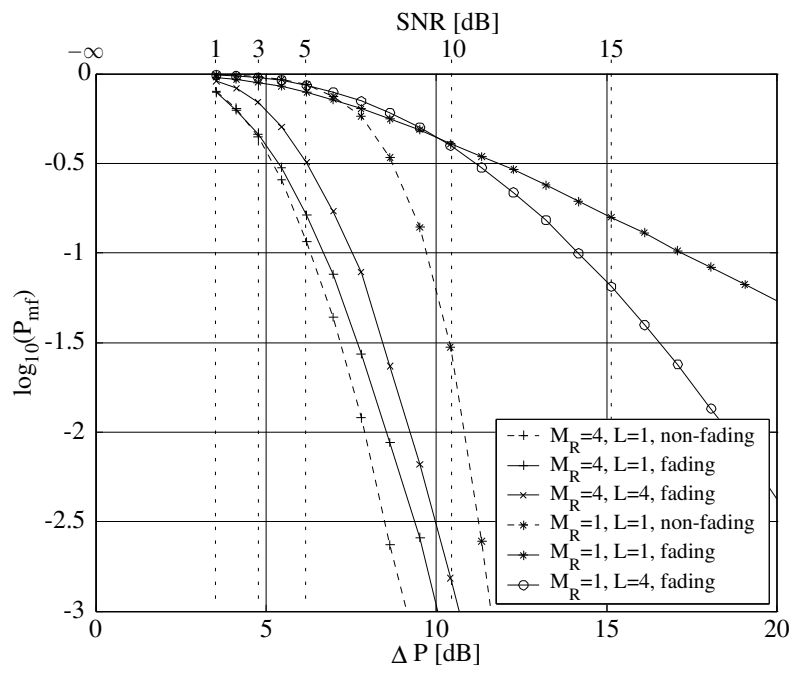

Fig. 4. Simulated missed frame rates $P_{\mathrm{mf}}$ at different receive SNRs, drawn as corresponding power increases $\Delta P$. Different channel scenarios in a $4 \times 4$ IEEE 802.11a based MIMO-OFDM system are considered. The false alarm rate is $10 \%$ and $W=8000$. On the top of this figure the corresponding $\mathrm{SNR}_{\mathrm{dB}}$ are drawn.

Discussion: As shown in Fig. 4, the peak-detection algorithm does not work well for a single receive antenna scenario. However, the miss frame probability quickly decreases for a larger number of receive antennas, e.g. $M_{R}=4$. There, the detector is able to exploit the degrees of freedom offered by multiple receive antennas. Shorter channels exhibit better $P_{\mathrm{mf}}$ as the power increase is more pronounced for a low number of taps. However, the proposed algorithm is also able to detect frame-starts even for a larger number of channel taps $L$. The performance is superior if no fading is present in the system, since the received signals never incur deep fades. The onetap non-fading scenario matches best with the $P_{\mathrm{mf}}$ analyzed in the previous section (cf. Fig. 3).

\section{IMPLEMENTATION RESULTS}

The presented frame-start detection algorithm has been implemented for an IEEE 802.11a-based MIMO-OFDM system 


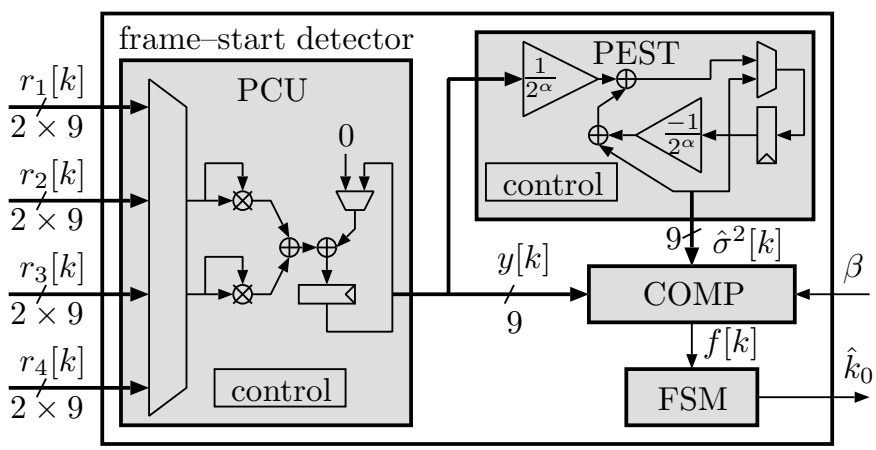

Fig. 5. Block diagram of the implemented frame-start detector. The power computation unit (PCU) produces $y[k]$ which is used by the PEST to obtain $\hat{\sigma}^{2}$. The comparator (COMP) compares $y[k]$ with $\beta \hat{\sigma}^{2}$ and the finite state machine (FSM) evaluates the detected peaks. Time sharing is employed in the PCU and an IIR-filter is employed in the PEST unit.

with four receive antennas. The throughput of the system corresponds to $20 \mathrm{Msps}$ and the target system clock rate is set to $80 \mathrm{MHz}$.

\section{A. Architecture}

The architecture of the frame-start detector has been implemented according to the block diagram depicted in Fig. 5 and consists of four main units.

a) Power Computation Unit (PCU): This block consists of two real-valued multipliers which are used to calculate the instantaneous power of all four receive antennas by means of time-sharing. An accumulator stage adds up all intermediate results in order to present a new $y[k]$ every fourth clock cycle. The time-sharing is controlled by control circuitry.

b) Power Estimator Unit (PEST): To lower the complexity of the circuit, the power estimator has been implemented as a simple infinite impulse response (IIR) filter:

$$
\hat{\sigma}^{2}[k]=\left(1-2^{-\alpha}\right) \hat{\sigma}^{2}[k-1]+2^{-\alpha} y[k-1],
$$

where $\alpha$ has been set to 7 and $\hat{\sigma}^{2}[-\infty]=1$. In order to prevent a quick increase in the average signal power estimate $\hat{\sigma}^{2}$ at a frame start, the IIR-filter is not updated if $y[k]>4 \hat{\sigma}^{2}$. This forces the filter to ignore samples with very high power. However, the signal power estimate $\hat{\sigma}^{2}$ is not severely affected as these peaks do not contribute significantly to the signal power estimate.

c) Comparator Unit (COMP): The comparator unit is able to use the following thresholds: $\beta=1,2,4,5,6,8,12,16$. Due to the large dynamic range of incoming signals in OFDM systems, 9-bit precision has been employed.

d) Finite State Machine (FSM): Upon the first reception of a peak (i.e., $f\left[\hat{k}_{0}\right]=1$ ), the state machine observes subsequent samples. If $\sum_{k=\hat{k}_{0}}^{\hat{k}_{0}+F} f[k]>T$, the FSM outputs the instant of the first detected peak as frame-start estimate $\hat{k}_{0}$. Here, both parameters $F$ and $T$ additionally reduce the false frame detection probability, while keeping $P_{\mathrm{mf}}$ virtually unchanged.

\section{B. Implementation Results}

The implementation results for a Virtex2 FPGA are shown in Tab. II. These results confirm the low-complexity architecture of the proposed frame-start detector:
TABLE II

FRAME-START DETECTOR IMPLEMENTATION ON AN VIRTEX2 FPGA FOR FOUR RECEIVE ANTENNAS. THE DESIGN RUNS AT $80 \mathrm{MHZ}$ WITH A SAMPLING RATE OF 20 MSPS.

\begin{tabular}{|l||c|c|c|}
\hline Unit & \#Slices & \#FF & \#Multipliers \\
\hline \hline PCU & 86 & 59 & 2 \\
\hline PEST & 23 & 16 & 0 \\
\hline COMP & 31 & 0 & 0 \\
\hline FSM & 14 & 15 & 0 \\
\hline \hline total & 154 & 90 & 2 \\
\hline
\end{tabular}

- Virtually no storage elements are needed as opposed to [2], where delayed samples are required to obtain the frame timing.

- Only two multipliers are needed in the proposed implementation, which would not suffice if optimized approaches (e.g. matched filters) would be employed.

Additionally, some components (such as the PEST and the PCU) can be shared with other synchronization stages, e.g. for a frequency offset estimator.

\section{CONCLUSIONS}

A low-complexity frame-start detection algorithm for MIMO systems has been presented. The algorithm only relies on instantaneous power increase and peak detection. The proposed detector is signal-property independent and thus, universally applicable to frame-based MIMO communication systems. The performance gain caused by the use of multiple receive antennas is shown both analytically and through simulations performed in a MIMO-OFDM system.

Applied to a $4 \times 4$ IEEE 802.11 a based MIMO-OFDM system, the algorithm shows a satisfactory frame-start detection performance above $10 \mathrm{~dB}$ SNR and has shown to be robust for fading, non-fading, and frequency-selective channels. Finally, the FPGA implementation has shown to be of low-complexity and thus, provides a viable solution for real-time frame-start detection.

\section{REFERENCES}

[1] A. Paulraj, R. Nabar, and D. Gore, Introduction to Space-Time Wireless Communications. Cambridge Univ. Press, 2003.

[2] T. M. Schmidl and D. C. Cox, "Robust frequency and timing synchronization for OFDM," IEEE Transactions on Communications, vol. 45, pp. 1613-1621, 1997.

[3] A. Fort and W. Eberle, "Synchronization and AGC proposal for IEEE 802.11 a burst OFDM systems," in Proc. IEEE GLOBECOM '03., vol. 3 , 2003, pp. 1335-1338.

[4] A. van Zelst and T. C. W. Schenk, "Implementation of a MIMO-OFDMBased wireless LAN system," IEEE Transactions on Signal Processing, vol. 52, pp. 483-494, 2004.

[5] D. Perels, S. Haene, A. Burg, P. Luethi, N. Felber, and W. Fichtner, "A frame-start detector for a 4 x 4 MIMO-OFDM system," in Proc. ICASSP'06, vol. 4, 2006, pp. 425-428.

[6] A. Papoulis and S. U. Pillai, Probability, Random Variables and Stochastic Processes, 4th ed. McGraw-Hill Book Co., 2002.

[7] Supplement to IEEE Standard for Information technology - Telecommunications and information exchange between systems - Local and metropolitan area networks - Specific requirements, Part 11: Wireless LAN Medium Access Control (MAC) and Physical Layer (PHY) specifications, Highspeed Physical Layer in the $5 \mathrm{GHz}$ Band, iSO/IEC 8802-11:1999/Amd 1:2000(E).

[8] D. Perels, A. Burg, S. Haene, N. Felber, and W. Fichtner, "An automatic gain controller for MIMO-OFDM WLAN systems," in Proc. IEEE ICCSC '06, vol. 1, 2006, pp. 55-60. 